\title{
Capacidade de Redes Ad Hoc sem Fio utilizando um Modelo de Canal com Desvanecimento Rayleigh e Atenuação com Distância
}

\author{
Altenis V. de Lima-e-Lima, Carlos E. B. Cruz Pimentel, Renato M. de Moraes
}

\begin{abstract}
Resumo-Este artigo apresenta o cálculo da capacidade de canal para redes ad hoc móveis (em inglês mobile ad hoc networks, MANETs) densas utilizando um modelo de comunicação que inclui atenuação com distância e desvanecimento Rayleigh. É mostrado que a capacidade não decai a zero quando o número (n) de nós na rede cresce ao infinito desde que o parâmetro de atenuação com a distância $(\alpha)$ seja maior que dois e que os nós transmissores procurem transmitir para nós receptores que estejam suficientemente próximos. Resultados analíticos e obtidos por simulação Monte-Carlo são comparados. É também proposta uma técnica de coordenação tal que os nós possam comportarse ora como transmissor ora como receptor de acordo com um parâmetro $(\theta)$ de fração de transmissores na rede.
\end{abstract}

Palavras-Chave-Capacidade, interferência, redes ad hoc.

Abstract-This paper presents the channel capacity computation for dense mobile Ad Hoc networks (MANETs) using a communication channel model which includes path loss and Rayleigh fading. It is shown that the capacity does not decay to zero when the total number of nodes $(n)$ in the network scales to infinity as long as the path loss parameter $(\alpha)$ is greater than two and a node attempts to transmit to a close neighbor. Analytical and Monte-Carlo simulation results are shown and compared. This work also proposes a coordination technique among nodes such that they either can be a senser or a receiver according to a parameter $(\theta)$ describing the fraction of senders in the network.

Keywords—Ad hoc networks, channel capacity, interference.

\section{INTRODUÇÃO}

Os efeitos da interferência restringem bastante o desempenho das redes ad hoc do mesmo modo que Gupta e Kumar [1] mostraram que a capacidade de uma determinada rede sem fio fixa diminui quando o número total de nós $n$ na rede aumenta. Mais especificamente, eles provaram que a capacidade do nó diminui aproximadamente com $1 / \sqrt{n}$. Grossglauser e Tse [2] apresentaram uma técnica de repasse de pacotes em duas fases para MANETs utilizando a diversidade multiusuário [3] na qual um nó fonte transmite um pacote para o vizinho mais próximo, e este, por sua vez, entrega o pacote ao destino quando este destino se torna seu vizinho mais próximo. O esquema demonstrou [2] melhorar a capacidade das MANETs, de modo que a mesma permaneça constante à medida que o número de usuários na rede aumenta, aproveitando-se do fato de que a comunicação entre nós próximos supera a interferência dos nós mais distantes.

A. V. de Lima-e-Lima e C. E. B. Cruz Pimentel estão no Departamento de Sistemas e Computação, Universidade de Pernambuco (UPE). E-mails: avll@dsc.upe.br, cebcp@dsc.upe.br

R. M. de Moraes está no Departamento de Engenharia Elétrica, Universidade de Brasília (UnB). E-mail: renatomdm@unb.br
Por outro lado, modelos detalhados e objetivos para o cálculo da interferência em redes $a d$ hoc densas não têm sido extensivamente estudados. Modelos de grade foram propostos para calcular a interferência [4], [5], os quais se beneficiam da disposição regular dos nós na rede. Esta topologia regular é um bom ponto de partida para redes estáticas, entretanto, não se aplica às MANETs. Da mesma forma, alguns trabalhos anteriores assumiram um alcance de transmissão ou de recepção para comunicação entre os nós sem considerar o efeito de toda a rede [6], [7]. Esta aproximação pode ser boa para redes de baixa densidade, mas pode implicar em resultados imprecisos para redes densas. Um problema com tal aproximação é a dificuldade em encontrar uma descrição analítica para a topologia aleatória inerente às redes $a d$ hoc. Em outros casos, modelos analíticos usam teoria de grafos [8], [9], que embora sejam bons para análises de camadas altas de rede, não são apropriados para um estudo mais detalhado do canal de comunicação que considerem parâmetros físicos como o fator de atenuação e o desvanecimento.

Neste artigo, é apresentado um modelo de canal de comunicação, a partir do modelo proposto por Moraes et al. [10] que não considerou desvanecimento, o qual permite obter a relação sinal-ruído e interferência (SNIR, do inglês signal-to-noise and interference ratio) medida por um nó receptor e, conseqüentemente, sua capacidade de canal (ou eficiência espectral) [11] em qualquer ponto na rede quando se comunica com um vizinho próximo. $\mathrm{O}$ estudo aqui descrito considera a distância Euclidiana, o fator de atenuação com a distância $(\alpha)$ e o desvanecimento Rayleigh [12]. É aqui admitido um modelo de mobilidade aleatória para os nós com o parâmetro $\theta$ representando a fração de nós transmissores na rede. Simulações Monte-Carlo [13] são apresentadas para validar o modelo analítico obtido. Diferentemente de trabalhos anteriores que assumiam o nó receptor localizado apenas no centro da rede [14], [15], [16], os resultados apresentados neste artigo são mais gerais, já que se referem a qualquer posição do nó na rede. Assim sendo, conclui-se que se um nó se comunica com seus vizinhos próximos quando o fator de atenuação $\alpha$ é maior que dois, a SNIR recebida e a capacidade de canal tendem a um valor constante à medida que $n$ aumenta, independentemente da posição do nó na rede, ou seja, esteja o nó receptor no centro, no meio, ou na borda da área da rede. Ainda para o caso onde $\alpha$ é igual a dois, a SNIR limite e a capacidade de canal decaem para zero quando $n$ cresce, entretanto, eles decaem lentamente, fazendo a comunicação local ainda possível para $n$ finito. 
Outra contribuição deste trabalho é a apresentação de uma técnica autônoma e distribuída entre os nós para determinação dos estados (transmissor ou receptor) de cada nó na rede em função do parâmetro $\theta$.

O restante do artigo é organizado como segue. A Seção II apresenta o modelo da rede. A Seção III mostra a relação do número médio de possíveis nós receptores vizinhos como uma função dos parâmetros da rede. A Seção IV explica o cálculo da interferência e da eficiência espectral. Os resultados analíticos e da simulação são comparados na Seção V. A Seção VI conclui o artigo fazendo um resumo dos principais resultados obtidos. No Apêndice os teoremas são demonstrados, como também é apresentada uma proposta para uma técnica autônoma e distribuída de determinação de estados de nós e descobrimento de vizinhança.

\section{MODELO}

O problema aqui tratado é de uma rede ad hoc sem fio com nós considerados móveis. Este modelo consiste de uma área circular unitária normalizada (ou disco) contendo $n$ nós e assemelha-se ao modelo de Grossglauser e Tse [2]. O fluxo de informação na rede segue a técnica de retransmissão de pacote em duas fases como descrito em [2]. A posição do nó $i$ no tempo $t$ é indicado por $X_{i}(t)$. Assume-se que os nós se movem de acordo com o modelo uniforme de mobilidade [17]. Este modelo satisfaz as seguintes propriedades [17]: (a) as posições dos nós são independentes umas das outras em qualuqer tempo t; (b) a distribuição dos nós móveis é uniforme; (c) a direção do movimento do nó é uniformemente distribuida em $[0,2 \pi)$, condicionado à posição do nó.

Uma fração do número total de nós $n$ na rede, $n_{S}$, é escolhido aleatoriamente como nós transmissores, enquanto os nós restantes, $n_{r}$, operam como possíveis nós receptores [2]. Um parâmetro de densidade de transmissores $\theta$ é definido por $n_{S}=\theta n$, onde $\theta \in(0,1)$ e $n_{R}=(1-\theta) n$. No Apêndice I é apresentada uma técnica de adequação da rede para um parâmetro $\theta$ desejado.

Um nó receptor $j$ no tempo $t$ é capaz de receber dados a uma dada taxa de tranmissão de $W$ bits/seg do nó $i$ se [2], [1]

$$
S N I R=\frac{P_{i}(t) g_{i j}(t)}{N_{0}+\frac{1}{L} \underbrace{\sum_{k \neq i} P_{k}(t) g_{k j}(t)}_{I}}=\frac{P_{i}(t) g_{i j}(t)}{N_{0}+\frac{1}{L} I} \geq \beta,
$$

onde $P_{i}(t)$ é a potência de transmissão do nó $i, g_{i j}(t)$ é o ganho do canal do nó $i$ ao nó $j, \beta$ é o nível necessário para uma comunicação confiável, $N_{o}$ é a densidade espectral da potência de ruído, $L$ é o ganho de processamento do sistema e $I$ é a interferência total no nó $j$. Assume-se que nenhum ganho de processamento é usado, ou seja, $L=1$, e que $P_{i}=P \forall i$. O ganho de canal é considerado como uma função da distância e do desvanecimento tal que [2], [1], [18]

$$
g_{i j}(t)=\frac{\chi_{i j}^{2}}{\left|X_{i}(t)-X_{j}(t)\right|^{\alpha}}=\frac{\chi_{i j}^{2}}{r_{i j}^{\alpha}}
$$

onde $\chi_{i j}^{2}$ é a variável aleatória que representa o desvanecimento Rayleigh do nó $i$ ao nó $j, r_{i j}$ é a distância Euclidiana entre estes nós, e $\alpha$ é o coeficiente de atenuação com a distância.
Este artigo visa encontrar uma equação relacionando a interferência total medida por um nó receptor que está se comunicando com um nó vizinho como uma função do número total $n$ de usuários na rede. Mais precisamente será obtida uma expressão para Eq. (1) como uma função de $n$ e calcular-se-á o limite de SNIR e, conseqüentemente, a capacidade de canal quando $n$ tende ao infinito.

\section{Possíveis Receptores Próximos A UM TRANSMISSOR}

A fim de se obter a interferência gerada por nós fora da vizinhança de um nó receptor, primeiro é preciso encontrar o tamanho médio do raio contendo um nó transmissor e seus possíveis receptores que estão nesta vizinhança.

Se a densidade dos nós no disco for

$$
\rho=\frac{n}{\text { total area }}=\frac{n}{1}=n,
$$

então o raio médio $\left(r_{o}\right)$ para um nó transmissor para uma distribuição uniforme de nós é dado por

$$
1=\theta \rho \pi r_{o}^{2}=\theta n \pi r_{o}^{2} \Longrightarrow r_{o}=\frac{1}{\sqrt{\theta n \pi}} .
$$

Portanto, o número médio de nós receptores, chamado de $\bar{K}$, dentro de $r_{o}$, assumindo uma distribuição uniforme de nós devido ao modelo de mobilidade empregado [17], é

$$
\bar{K}=n_{R} \pi r_{o}^{2}=(1-\theta) n \pi\left(\frac{1}{\sqrt{\theta n \pi}}\right)^{2}=\frac{1}{\theta}-1,
$$

o qual é constante para um dado $\theta$. A Eq. (5) é uma referência prática para obter o número médio dos nós receptores como uma função do parâmetro $\theta$ da rede .

Assim, o raio $r_{o}$ define uma célula (alcance do raio) ao redor de um transmissor, onde, na média, $\bar{K}$ receptores estão próximos. A possibilidade de todos estes $\bar{K}$ nós receberem com sucesso os mesmos dados sendo transmitidos pelo transmissor é o assunto para a próxima seção.

\section{INTERFERÊNCIA E CÁlCULO DA CAPACIDADE}

$\mathrm{Na}$ seção anterior foi obtido o raio médio $r_{o}$ contendo um transmissor com $\bar{K}$ nós receptores ao redor, em média. Supondo que um dos $\bar{K}$ nós receptores está nas proximidades de $r_{o}$, quer-se saber como a SNIR medida por este receptor se comporta à medida que o número dos nós totais na rede (e assim o número de interferentes totais) vai para o infinito. É importante determinar se ainda é possível uma comunicação viável entre o transmissor e o vizinho mais distante ${ }^{1}$ (distando $r_{o}$ ), mesmo que o número de interferentes aumente.

A Eq. (1) deve ser satisfeita para que um pacote seja recebido com sucesso. Portanto, considere um receptor em qualquer local na rede em um dado tempo $t$. Sua distância $r^{\prime}$ ao centro é mostrada na Fig. 1 , onde $0 \leq r^{\prime} \leq \frac{1}{\sqrt{\pi}}-r_{o}$.

Assumindo que o transmissor está situado a uma distância $r_{o}$ deste receptor e transmitindo à potência constante $P$, então a potência $P_{o}$ medida por este receptor é dada por

\footnotetext{
${ }^{1}$ Isto representa o pior caso, porque os outros $\bar{K}-1$ vizinhos estão localizados mais próximos ou na mesma distância $r_{o}$ do transmissor, assim eles medem um valor de SNIR mais forte ou o mesmo valor.
} 


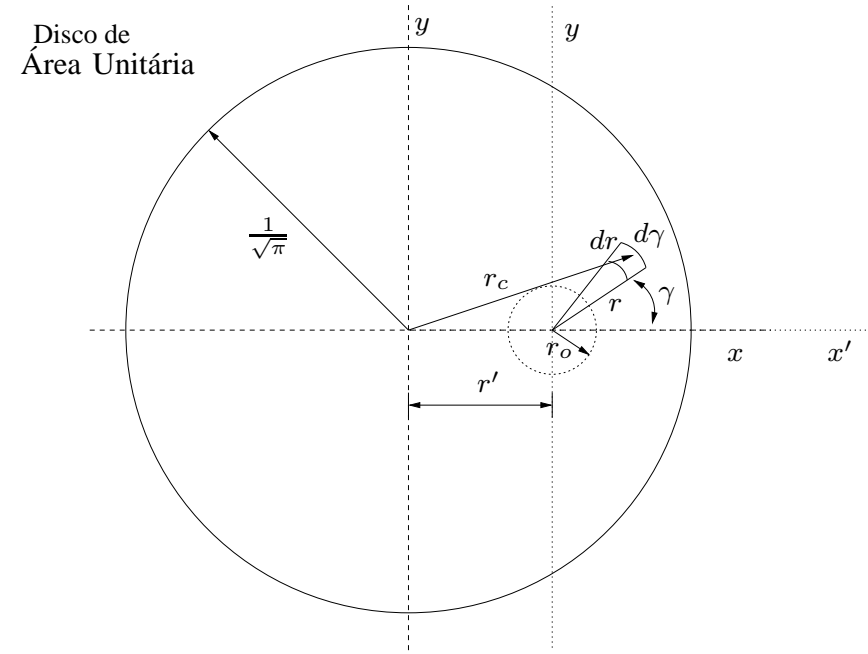

Fig. 1. Disco de área unitária em um dado tempo $t$. Neste momento, o nó receptor sendo analisado está localizado à distância $r^{\prime}$ do centro, enquanto o transmissor está a uma distância $r_{o}$ do nó receptor.

$$
P_{o}=\frac{P \chi_{o}^{2}}{r_{o}^{\alpha}}
$$

onde $\chi_{o}^{2}$ é o desvanecimento Rayleigh do transmissor para o receptor.

Com a finalidade de obter a interferência total média no receptor causada por todos os nós transmissores, considere um elemento diferencial de área $r d r d \gamma$ que está distante $r$ unidades do receptor e $r_{c}$ unidades do centro da rede (veja Fig. 1). Como conseqüência do modelo de mobilidade uniforme, a distribuição dos nós é uniforme [17]. Assim, a função de densidade de probabilidade da distância $r_{c}$ ao centro da rede é dada por [14]

$$
f_{R_{c}}\left(r_{c}\right)= \begin{cases}2 \pi r_{c} & \text { se } 0 \leq r_{c} \leq \frac{1}{\sqrt{\pi}} \\ 0 & \text { caso contrário. }\end{cases}
$$

Já que os nós estão distribuidos de forma uniforme no disco, os nós transmissores dentro do elemento diferencial de área geram, no receptor, a seguinte interferência ${ }^{2}$

$$
d I=\frac{P \chi^{2}}{r^{\alpha}} \theta \rho r d r d \gamma=\frac{P \chi^{2}}{r^{\alpha-1}} \theta n d r d \gamma .
$$

A interferência total é obtida integrando a Eq. (8) na área do disco e o resultado depende do valor de $\alpha$. Conseqüentemente, os dois casos a seguir são considerados.

\section{A. $O$ caso $\alpha>2$}

Para alguns ambientes de propagação [12, p. 139, Tabela 4.2] o fator de atenuação é modelado para ser sempre maior que dois, ou seja, $\alpha>2$. Neste caso, a SNIR no receptor localizado à distância $r^{\prime}$ do centro para um total de $n$ nós na rede é dado no teorema seguinte.

Teorema 1: Num dado tempo $t$, para um nó receptor localizado à distância $r^{\prime}$ do centro em uma rede circular de área unitária contendo $n$ nós móveis uniformemente distribuidos, onde $\alpha>2$, e considerando o transmissor localizado à

\footnotetext{
${ }^{2}$ Já que os nós estão uniformemente distribuidos no disco e $n$ cresce para o infinito, a soma na Eq. (1) foi aproximada por uma integral.
}

distância $r_{o}$ deste receptor, então a SNIR no receptor é dada por

$$
\operatorname{SNIR}_{r^{\prime}}(n)=\frac{P e^{\delta \sigma_{s}}}{\frac{N_{0}}{(\theta n \pi)^{\frac{\alpha}{2}}}+\frac{2 P e^{\delta \sigma_{s}}}{\alpha-2} \cdot q_{r^{\prime}, \alpha, \theta}(n)},
$$

onde $\delta=\frac{\ln (10)}{10}, \sigma_{s}$ é o desvio padrão da variável aleatória Gaussiana da atenuação em decibeis devido ao sombreamento [18], e

$$
q_{r^{\prime}, \alpha, \theta}(n)=\left[1-\frac{\int_{0}^{\pi}\left[\sqrt{\frac{1}{\pi}-\left(r^{\prime} \sin \gamma\right)^{2}}-r^{\prime} \cos \gamma\right]^{2-\alpha} d \gamma}{\pi^{\frac{\alpha}{2}}(\theta n)^{\frac{\alpha-2}{2}}}\right]^{-1} .
$$

Prova: Ver Apêndice II.

Da Eq. (9), considerando o limite quando $n \rightarrow \infty$, obtem-se que

$$
\begin{aligned}
\text { SNIR } & =\lim _{n \rightarrow \infty} \frac{P e^{\delta \sigma_{s}}}{\frac{N_{0}}{(\theta n \pi)^{\frac{\alpha}{2}}}+\frac{2 P e^{\delta \sigma_{s}}}{\alpha-2} \cdot q_{r^{\prime}, \alpha, \theta}(n)} \\
& =\left\{\begin{array}{cc}
\frac{\alpha-2}{2} & \text { if } 0 \leq r^{\prime}<\frac{1}{\sqrt{\pi}}-r_{o} \\
\frac{\alpha-2}{2} q_{r^{\prime}, \alpha, \theta}(n \rightarrow \infty) & \text { if } r^{\prime}=\frac{1}{\sqrt{\pi}}-r_{o}, \text { i.e., }(11) \\
\text { a borda da rede. }
\end{array}\right.
\end{aligned}
$$

Da Eq. (10), $q_{r^{\prime}, \alpha, \theta}(n \rightarrow \infty)=q_{r^{\prime}, \alpha}(n \rightarrow \infty)$ visto que $\theta$ é um fator de escala de $n$ e não modifica o limite. Assim,

$$
q_{r^{\prime}, \alpha, \theta}(n \rightarrow \infty)= \begin{cases}1 & \text { se } 0 \leq r^{\prime}<\frac{1}{\sqrt{\pi}}-r_{o} \text { e } \alpha>2 \\ 1.47 & \text { se } r^{\prime}=\frac{1}{\sqrt{\pi}}-r_{o} \text { e } \alpha=3 \\ 1.33 & \text { se } r^{\prime}=\frac{1}{\sqrt{\pi}}-r_{o} \text { e } \alpha=4 \\ 1.27 & \text { se } r^{\prime}=\frac{1}{\sqrt{\pi}}-r_{o} \text { e } \alpha=5 \\ 1.23 & \text { se } r^{\prime}=\frac{1}{\sqrt{\pi}}-r_{o} \text { e } \alpha=6\end{cases}
$$

Portanto, das Eqs. (11) e (12), para $\alpha>2$, a SNIR tende a uma constante quando $n \rightarrow \infty$.

Do Teorema 1, a capacidade de canal $C$ (em unidades de bits/s/Hz) é obtida diretamente por [11]

$C=\log _{2}\left[1+S N I R_{r^{\prime}}(n)\right]=\log _{2}\left[1+\frac{P e^{\delta \sigma_{s}}}{\frac{N_{0}}{(\theta n \pi)^{\frac{\alpha}{2}}}+\frac{2 P e^{\delta \sigma_{s}}}{\alpha-2} q_{r^{\prime}, \alpha, \theta}(n)}\right]$

Conseqüentemente, das Eqs. (9), (12) e (13), conclui-se que a eficiência espectral tende a uma constante quando $n \rightarrow \infty$ e $\alpha>2$.

\section{B. $O$ caso $\alpha=2$}

Para o ambiente de propagação em espaço livre [12, p. 107], o fator de atenuação é modelado para ser igual a 2, ou seja, $\alpha=2$. Assim, a SNIR no receptor localizado à distância $r^{\prime}$ do centro para um total de $n$ nós na rede é obtido pelo teorema a seguir, cuja prova é análoga ao Teorema 1.

Teorema 2: Em um dado tempo $t$, para um nó receptor localizado à distância $r^{\prime}$ do centro em uma rede circular de área unitária contendo $n$ nós móveis uniformemente distribuidos, onde $\alpha=2$, e assumindo o transmissor localizado à distância $r_{o}$ deste receptor, então a SNIR no receptor é dada por $\operatorname{SNIR}_{r^{\prime}}(n)=\frac{P e^{\delta \sigma_{s}}}{\frac{N_{0}}{\pi n \theta}+\frac{2 P e^{\delta \sigma_{s}}}{\pi} \int_{0}^{\pi} \ln \left[(\sqrt{\pi \theta n})\left(\sqrt{\frac{1}{\pi}-\left(r^{\prime} \sin \gamma\right)^{2}}-r^{\prime} \cos \gamma\right)\right] d \gamma}$

Sendo assim, a capacidade de canal $C$ (em unidades de bits/s/Hz) é dada por [11]

$C=\log _{2}\left[1+\frac{P e^{\delta \sigma_{s}}}{\frac{N_{0}}{\pi n \theta}+\frac{2 P e^{\delta \sigma_{s}}}{\pi} \int_{0}^{\pi} \ln \left[(\sqrt{\pi \theta n})\left(\sqrt{\frac{1}{\pi}-\left(r^{\prime} \sin \gamma\right)^{2}}-r^{\prime} \cos \gamma\right)\right] d \gamma}\right]$

Da Eq. (14), fica claro que $S N I R_{r^{\prime}}(n) \rightarrow 0$ quando $n \rightarrow \infty$. Portanto, a eficiência espectral vai para zero quando $n \rightarrow \infty$ para $\alpha=2$. 


\section{Resultados}

Nesta seção, os resultados analíticos obtidos na seção anterior são comparados com simulações Monte-Carlo [13]. A Fig. 2 mostra a capacidade como uma função de $n$ para $\alpha=3$, $\theta=\frac{1}{3}$, para diferentes valores de $r^{\prime}$. Além disso, a Fig. 2 demostra que a capacidade permanece constante quando $n$ vai para o infinito e esta constante não depende de $r^{\prime}$ se $0 \leq r^{\prime}<\frac{1}{\sqrt{\pi}}-r_{o}$, isto é, ela é o mesmo valor para qualquer posição do nó receptor dentro do disco, seja o centro, próximo a borda, ou no meio da região do raio do disco.

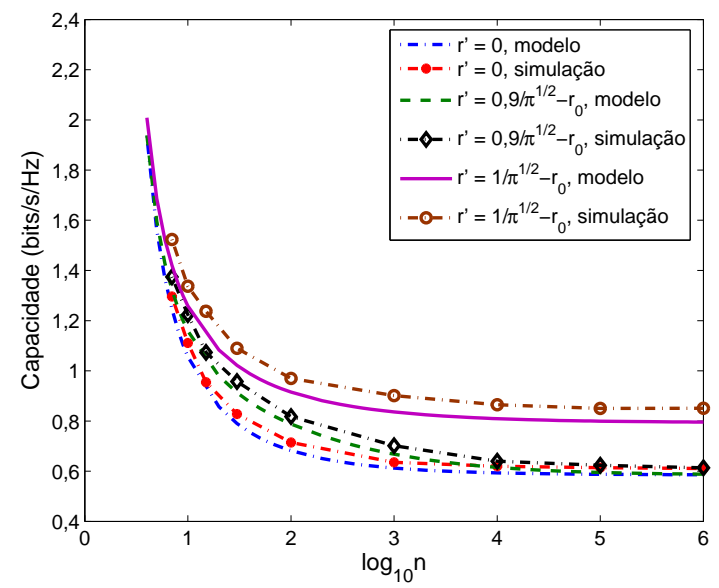

Fig. 2. Capacidade em função do número de nós $(n)$ para $\alpha=3, \theta=\frac{1}{3}$, $P=1 W, N_{0}=5$ e $\sigma_{s}=6$, para o nó receptor localizado no centro $\left(r^{\prime}=0\right)$ e na borda da rede (demais casos indicados).

A Fig. 3 mostra que o comportamento da capacidade para diferentes valores de $N_{o}$ quando $P=1 \mathrm{~W}$, e que ela diminui quando o ruído aumenta, mas a capacidade limite (quando $n \rightarrow \infty$ ) é a mesma independente do valor do ruído visto que a interferência é o termo dominante no denominador da Eq. (1) quando $n \rightarrow \infty$.

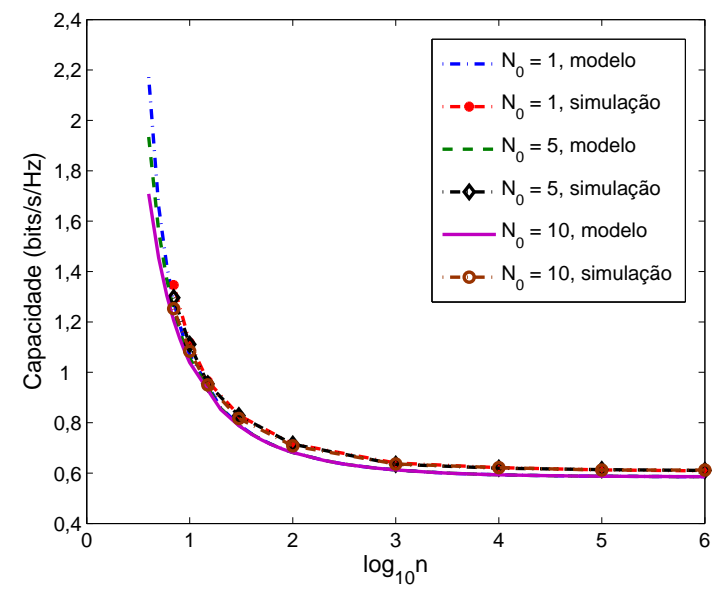

Fig. 3. Capacidade em função do número de nós $(n)$ para $\alpha=3, \theta=\frac{1}{3}$, $P=1 W, \sigma_{s}=6$, para diferentes valores de $N_{0}$.

A Fig. 4 confirma que a capacidade limite não depende do parâmetro $\theta$ conforme demonstrado na seção anterior.

A Fig. 5 mostra a capacidade em função do número de nós (n) para diferentes valores de $\sigma_{s}$ quando $\alpha=3$.

A Fig. 6 mostra a capacidade como uma função de $n$ para $\alpha=2$. Pode-se observar, conforme previsto no Teorema 2 ,

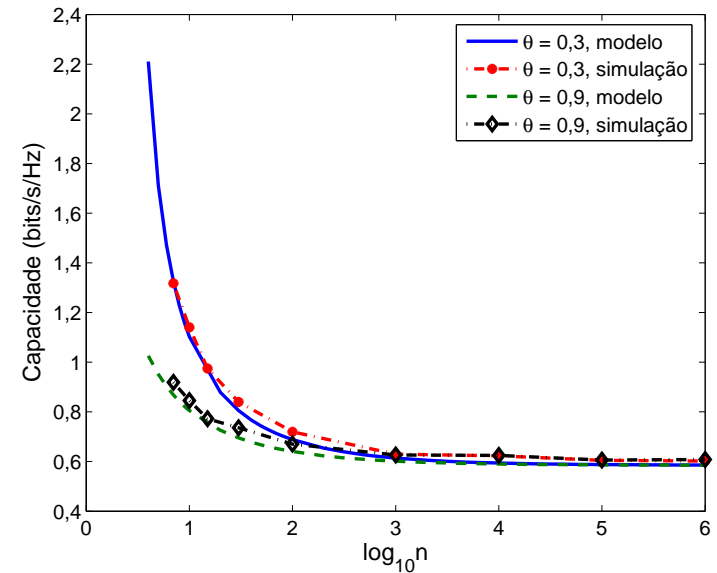

Fig. 4. Capacidade em função do número de nós $(n)$ para $\alpha=3, P=1 W$, $N_{0}=5, \sigma_{s}=6$, para diferentes valores de $\theta$.

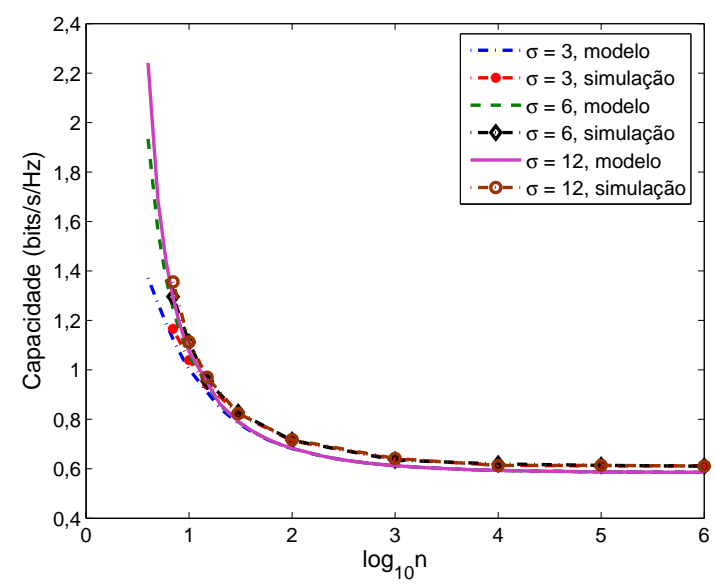

Fig. 5. Capacidade em função do número de nós $(n)$ para $\alpha=3, P=1 W$, $N_{0}=5, \theta=\frac{1}{3}$, com diferentes valores de $\sigma_{s}$.

que a capacidade decai a zero, entretanto o faz lentamente, tornando ainda possível a comunicação para $n$ finito dependendo do valor de $\beta$ na Eq. (1).

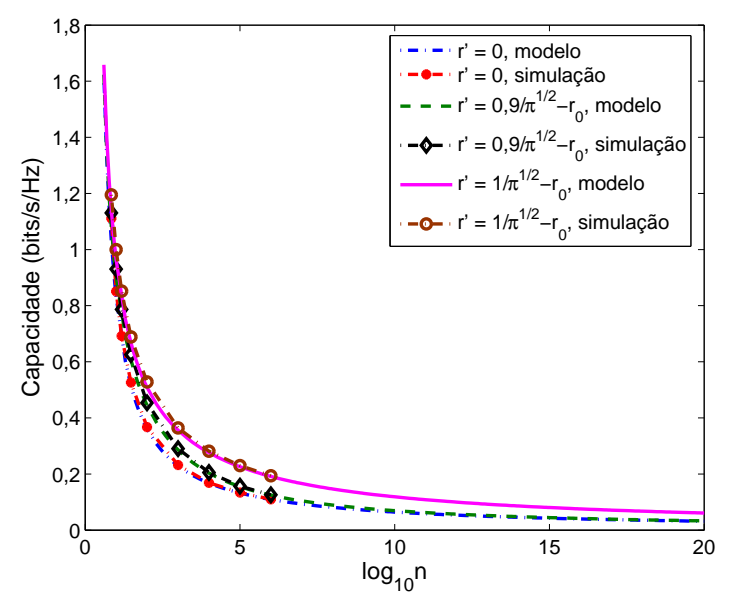

Fig. 6. Capacidade em função do número de nós $(n)$ para $\alpha=2, \theta=\frac{1}{3}$, $P=1 W, N_{0}=5$ e $\sigma_{s}=6$ 


\section{Conclusões}

Foram analisados os efeitos da interferência e da capacidade de canal para redes ad hoc móveis usando um modelo de canal de comunicação, considerando distância Euclidiana e desvanecimento Rayleigh empregando-se mobilidade aleatória para os nós. Este estudo mostra que, para um nó receptor se comunicando com um vizinho próximo, onde o fator de atenuação $(\alpha)$ é maior que dois, a relação sinal ruído e interferência (SNIR) e conseqüentemente a capacidade de canal, tendem a um valor constante quando o número total de nós $n$ cresce ao infinito, independente da posição do nó receptor na rede. Portanto, a comunicação é possível para vizinhos próximos quando o número de interferentes aumenta. Além disso, para os nós receptores localizados na borda da rede circular, verifica-se que eles sofrem menos interferência que aqueles localizados mais internamente, alcançando uma maior capacidade. Também para o caso onde $\alpha$ é igual a dois, foi mostrado que a capacidade vai para zero à medida que $n$ aumenta; entretando, o decaimento é muito lento tornando a comunicaçao local ainda possível para um $n$ finito. Também foi apresentada uma proposta para uma técnica autônoma e distribuída de determinação de estados de nós e descobrimento de vizinhança na rede. Trabalhos futuros podem considerar tratamento de colisão nos ciclos de contenda, como também controle de potência e outros modelos de mobilidade (que resultem em outras distribuições de nós na rede), e como eles afetam a capacidade.

\section{APÊNDICE I}

\section{TÉCNICA PARA AdEQUAÇÃo DA REDE AO PARÂMETRO $\theta$}

Outro desafio associado a este estudo é como um nó deve determinar seu estado na rede, i.e., se atua como transmissor (Tx) ou receptor $(\mathrm{Rx})$ num dado ciclo de comunicação. Os trabalhos apresentados por Grossglauser e Tse [2], e complementados por Moraes et al. [19], demonstram analiticamente e por meio de simulação, que a máxima vazão, para uma rede $a d$ hoc sem fio, é alcançada quando a fração de nós transmissores $(\theta)$ está próximo de $\frac{1}{3}$ do total de nós existentes na rede. Porém, não há estudos na literatura acerca de protocolos da camada de acesso ao meio (MAC, do inglês medium access control) que busquem esta distribuição de maneira autônoma e distribuída.

A técnica aqui sugerida para se atingir um dado $\theta$ na rede, consiste em um modelo simplificado de parte de um protocolo MAC. Tal como o TRAMA [20], tem o requisito de ser sincronizado e com períodos cíclicos de contenda e transmissão de dados, e a capacidade de alguns nós assumirem o controle dos demais dentro de seu raio de transmissão, tal como verificado no IEEE 802.15.4 - ZigBee [21]. Esta técnica, ainda restrita somente ao período de contenda, destina-se à determinação autônoma e distríbuida dos estados dos nós, a partir do parâmetro $\theta$.

Assume-se também que a distribuição dos nós é conforme o modelo descrito na Seção II, e que cada nó tem idenficação (ID) única na rede. O nó com menor ID assume o controle da rede.
Um ciclo de comunicação entre os nós consiste em dois períodos: o período de contenda e o período de transmissão. O período de contenda é dividido em três fases, respectivamente. A fase de anúncio, onde cada nó divulga por difusão o seu ID. A fase de comparação, onde os nós comparam os IDs divulgados para determinar quem é o nó controlador. $\mathrm{E}$ finalmente a fase de distibuição, onde o nó controlador divulga por difusão uma seqüência aleatória indicando o estado que cada nó na rede deve assumir (se Tx ou Rx) durante o periodo de transmissão, de acordo com o parâmetro $\theta$ previamente programado.

Na fase de distribuição, o nó controlador forma a seqüência aleatória (vetor) de $n$ posições, contendo os estados dos $n$ nós da rede, inclusive ele mesmo o controlador, onde cada posição armazena um bit com valor 0 (para o estado receptor) ou 1 (para o estado transmissor), de acordo com o $\theta$ programado. A primeira posição desta seqüência correspondente ao nó com menor número de identificação, e última posição ao nó com maior número de identificação divulgados. Por exemplo, se houver um total de 100 nós na rede, o nó controlador divulgará um vetor contendo 100 posições, das quais $\lceil 100 \times \theta\rceil$ conterão bits 1 e as restantes bits 0 , aleatoriamente distribuídos, onde $\lceil x\rceil$ significa o seguinte inteiro maior ou igual a $x$.

Para determinar seu estado, cada nó ao receber a seqüência do nó controlador, verifica qual o valor do bit armazenado na posição correspondente ao seu ID. O nó permanece neste estado durante todo o ciclo de transmissão seguinte, e a cada ciclo de contenda as três fases são repetidas.

A Fig. 7 apresenta o resultado de uma simulação em linguagem JAVA [22], usando o método shuffle da classe Collections [23] para a distribuição aleatória dos estados Tx e Rx, onde estão exibidos a fração de vezes que três nós escolhidos ao acaso foram transmissores ao longo de 100 ciclos, para $\theta=\frac{1}{3}$ e $n=1000$. Observa-se que ao longo dos ciclos a fração que cada nó foi Tx converge para o valor desejado de $\theta$.

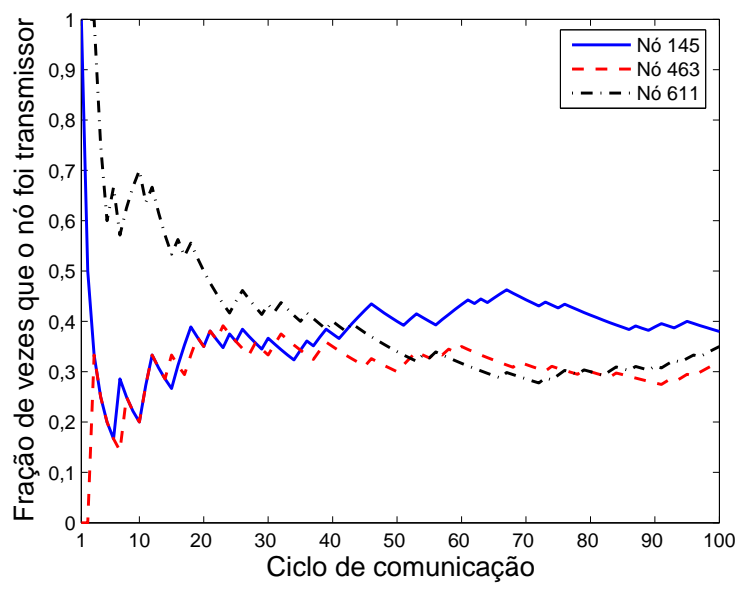

Fig. 7. Gráfico da evolução da fração de vezes que três nós escolhidos aleatoriamente foram Tx ao longo dos ciclos simulados, para $n=1000$, considerando-se $\theta=\frac{1}{3}$.

Esta técnica e simulação ainda não considerou o tratamento e controle da admissão e remoção dos nós, a resolução das 
possíveis colisões na fase de anúncio dos nós, o tamanho do período dos ciclos de contenda e transmissão, e nem o tamanho máximo da seqüência de estados. Estes aspectos serão objeto de estudos futuros.

\section{APÊNDICE II \\ PROVA DO TEOREMA 1}

Integrando a Eq. (8) sobre a área do disco, para $\alpha>2$, obtem-se a interferência no receptor localizado à distância $r^{\prime}$ do centro para um total de $n$ nós na rede. Assim,

$$
\begin{aligned}
E\left[I_{r^{\prime}}(n)\right] & =\int_{\text {disk area }} d I=E\left[\int_{0}^{2 \pi} \int_{r_{o}}^{r_{m}\left(r^{\prime}, \gamma\right)} \frac{P \chi^{2}}{r^{\alpha-1}} \theta n d r d \gamma\right] \\
& =\left.P e^{\delta \sigma_{s}} \theta n \int_{0}^{2 \pi} \frac{r^{2-\alpha}}{2-\alpha}\right|_{r_{o}} ^{r_{m}\left(r^{\prime}, \gamma\right)} d \gamma \\
& =\frac{P e^{\delta \sigma_{s}} \theta n}{\alpha-2} \int_{0}^{2 \pi}\left\{\frac{1}{r_{o}^{\alpha-2}}-\frac{1}{\left[r_{m}\left(r^{\prime}, \gamma\right)\right]^{\alpha-2}}\right\} d \gamma .
\end{aligned}
$$

$r_{m}$ é o máximo valor que $r$ terá em função da localização de $r^{\prime}$ e o ângulo $\gamma$ (veja Fig. 1). Para esta função, pode-se usar a equação da circunferência (borda da rede) em função dos eixos $x$ e $y$ apresentados em 1, i.e.,

$$
x^{2}+y^{2}=\left(\frac{1}{\sqrt{\pi}}\right)^{2} .
$$

Seja $x=x^{\prime}+r^{\prime}, x^{\prime}=r_{m} \cos \gamma$, e $y=r_{m} \sin \gamma$. Então, Eq. (17) será

$$
\begin{gathered}
\left(r_{m} \cos \gamma+r^{\prime}\right)^{2}+\left(r_{m} \sin \gamma\right)^{2}=\left(\frac{1}{\sqrt{\pi}}\right)^{2} \\
\Longrightarrow r_{m}\left(r^{\prime}, \gamma\right)=\sqrt{\frac{1}{\pi}-\left(r^{\prime} \sin \gamma\right)^{2}}-r^{\prime} \cos \gamma .
\end{gathered}
$$

Pela substituição deste resultado na Eq. (16), chega-se a

$$
E\left[I_{r^{\prime}}(n)\right]=\frac{2 P e^{\delta \sigma_{s}} \theta n}{\alpha-2}\left[\frac{\pi}{r_{o}^{\alpha-2}}-f_{\alpha}\left(r^{\prime}\right)\right],
$$

onde

$$
f_{\alpha}\left(r^{\prime}\right)=\int_{0}^{\pi} \frac{d \gamma}{\left[\sqrt{\frac{1}{\pi}-\left(r^{\prime} \sin \gamma\right)^{2}}-r^{\prime} \cos \gamma\right]^{\alpha-2}}
$$

é uma constante para qualquer posição $r^{\prime}$. Para o caso de $\alpha=4$, a Eq. (20) reduz-se

$$
f_{4}\left(r^{\prime}\right)=\frac{\pi^{2}}{1-2 \pi r^{\prime 2}+\pi^{2} r^{\prime 4}} .
$$

Pode-se obter a SNIR usando as Eqs. (1), (4), (6), e (19) para chegar em

$$
\begin{aligned}
\operatorname{SNIR}_{r^{\prime}}(n) & =\frac{E\left[P_{o}\right]}{N_{0}+E\left[I_{r^{\prime}}(n)\right]} \\
& =\frac{P e^{\delta \sigma_{s}}}{\frac{N_{0}}{(\theta n \pi)^{\frac{\alpha}{2}}}+\frac{2 P e^{\delta \sigma_{s}}}{\alpha-2} \cdot \frac{1}{1-\frac{1}{\pi^{\frac{\alpha}{2}}(\theta n) \frac{\alpha-2}{2}} f_{\alpha}\left(r^{\prime}\right)}} \\
& =\frac{P e^{\delta \sigma_{s}}}{\frac{N_{0}}{(\theta n \pi)^{\frac{\alpha}{2}}}+\frac{2 P e^{\delta \sigma_{s}}}{\alpha-2} \cdot q_{r^{\prime}, \alpha, \theta}(n)}, \\
\text { onde } \quad q_{r^{\prime}, \alpha, \theta}(n) & =\left[1-\frac{1}{\pi^{\frac{\alpha}{2}}(\theta n)^{\frac{\alpha-2}{2}}} f_{\alpha}\left(r^{\prime}\right)\right]^{-1} .
\end{aligned}
$$

\section{AGRADECIMENTOS}

Este trabalho foi financiado em parte pela Fundação de Amparo à Ciência e Tecnologia do Estado de Pernambuco (FACEPE), pela Escola Politécnica de Pernambuco, e pelo Conselho Nacional de Desenvolvimento Científico e Tecnológico (CNPq).

\section{REFERÊNCIAS}

[1] P. Gupta and P. R. Kumar, "The capacity of wireless networks,'IEEE Transactions on Information Theory, vol. 46, no. 2, pp. 388-404, March 2000.

[2] M. Grossglauser and D. Tse, "Mobility increases the capacity of wireless ad-hoc networks,"in Proc. of IEEE Infocom, Anchorage, Alaska, March 2001.

[3] R. Knopp and P. A. Humblet, 'Information capacity and power control in single-cell multiuser communications,"in Proc. of IEEE ICC, Seattle, Washington, June 1995.

[4] S. Gobriel, R. Melhem, and D. Mossé, "A unified interference/collision analysis for power-aware ad hoc networks,"in Proc. of IEEE Infocom, Hong Kong, March 2004.

[5] X. Liu and M. Haenggi, "Performance analysis of rayleigh fading ad hoc networks with regular topology,"in Proc. of IEEE Globecom, St. Louis, MO, November 2005.

[6] F. Tobagi and L. Kleinrock, "Packet switching in radio channels: Part ii - the hidden terminal problem in carrier sense multiple-access and the busy-tone solution,'IEEE Transactions on Communications, vol. COM23, no. 12, pp. 1417-1433, December 1975.

[7] J. Deng, Y. S. Han, P.-N. Chen, and P. K. Varshney, "Optimum transmission range for wireless ad hoc networks,"in Proc. of IEEE WCNC, Atlanta, GA, March 2004.

[8] P. von Rickenbach, S. Schmid, R. Wattenrofer, and A. Zollinger, "A robust interference model for wireless ad-hoc networks,"in Proc. of IEEE WMAN, Denver, CO, April 2005.

[9] D. Qin-yun, H. Xiu-lin, Y. Hong-yi, and Z. Jun, "On the capacity of wireless ad-hoc network basing on graph theory,"Lecture Notes in Computer Science, vol. 3421/2005, pp. 353-360, 2005.

[10] de Moraes, R. M., Buarque, Carlos Eduardo, ARAUJO, F. P., "Shannon Capacity for a Simple Communication Channel Model in Dense MANETs,"in Proc. of IEEE International Wireless Communications and Mobile Computing Conference (IWCMC), Creete, Grece, 2008.

[11] T. M. Cover and J. A. Thomas, Elements of Information Theory. John Wiley \& Sons, 1991.

[12] T. S. Rappaport, Wireless Communications: Principles and Practice. Prentice Hall, 2002.

[13] C. P. Robert and G. Casella, Monte Carlo Statistical Methods. SpringerVerlag, 2004.

[14] C. T. Lau and C. Leung, "Capture models for mobile packet radio networks,"IEEE Transactions on Communications, vol. 40, no. 5, pp. 917-925, May 1992.

[15] T. J. Shepard, "A channel access scheme for large dense packet radio networks,"in Proc. of ACM SigComm, San Francisco, California, August 1996.

[16] B. Hajek, A. Krishna, and R. O. LaMaire, "On the capture probability for a large number of stations,'IEEE Transactions on Communications, vol. 45, no. 2, pp. 254-260, February 1997.

[17] N. Bansal and Z. Liu, "Capacity, delay and mobility in wireless ad-hoc networks,"in Proc. of IEEE Infocom, San Francisco, California, March 2003.

[18] R. Akl, M. Hedge, M. Naraghi-Pour, and P. Min, "Multicell CDMA Network Design,'in IEEE Transactions on Vehicular Technoogy, vol. 50, no. 3, pp. 711-722, May 2001.

[19] de Moraes, R. M., Sadjadpour, H., and Garcia-Luna-Aceves, J. J., ’Taking full advantage of multiuser diversity in mobile ad hoc networks."in IEEE Transactions on Communications. June 2007.

[20] V. Rajendran, K. Obraczka, and J. J. Garcia-Luna-Aceves, "EnergyEfficient, Collision-Free Medium Access Control for Wireless Sensor Networks,'In Proc. of ACM SenSys 03, Los Angeles, CA, November 2003.

[21] ZigBee Alliance, "IEEE 802.15.4, ZigBee standard", http://www.zigbee.org/.

[22] Java Language, http://java.sun.com/, 2009

[23] Java Class Collections, http://java.sun.com/j2se/1.3/docs/api/java/util/Collections.html/, 2009 